\title{
Los directores(as) de las escuelas primarias rurales como los principales maestros de sus subordinados
}

\author{
Maria Julia Aguilar Aguilera ${ }^{1}$ \\ ${ }^{1}$ Universidad de Artemisa. Departamento superación de cuadros. Calle 8 C no 713 entre 7 y campo Consejo \\ Popular Lincoln. Artemisa - Cuba. mariajulia@uart.edu.cu
}

RESUMEN. El presente trabajo es el resultado de la labor de la autora como entrenadora de cuadros en la Universidad de Artemisa, el mismo tiene como objetivo proponer un sistema de acciones de capacitación para potenciar las habilidades de los directores (as) de las escuelas primarias rurales del municipio de Bauta como los principales maestros de sus subordinados. Para ello se asumió la capacitación desde la Universidad como actividad de postgrado en el puesto de trabajo, para favorecer el desarrollo de la actividad pedagógica profesional de dirección en el sistema de trabajo de la escuela. La utilización de diferentes métodos entre ellos la encuesta, la entrevista, la observación participante y el análisis documental permitió caracterizar el estado inicial, fundamentar la propuesta y valorar los resultados de su aplicación, lográndose generalmente que los directores asumieran el papel de maestro de sus subordinados.

Palabras clave: Sistema de Acciones, Capacitación, Actividad Pedagógica Profesional de Dirección y Escuelas Primarias Rurales. 


\title{
The directors of the rural primary schools as the main teachers of their subordinates
}

\begin{abstract}
The present work is the result of the work of the author as a trainer of paintings at the University of Artemisa. The aim of this work is to propose a system of training actions to enhance the skills of the principals of rural primary schools municipality of Bauta as the main teachers of his subordinates. To this end, the training was assumed from the University as a postgraduate activity in the workplace, in order to favor the development of the professional pedagogical activity of management in the school work system. The use of different methods such as the survey, interview, participant observation and documentary analysis allowed characterizing the initial state, substantiating the proposal and evaluating the results of its application, generally making it possible for the directors to assume the role of teacher of their subordinates.
\end{abstract}

Keywords: System of Actions, Training, Professional Pedagogical Activity of Management and Rural Primary Schools. 


\section{Os diretores(as) das escolas primárias rurais como principais professores de seus subordinados}

RESUMO. O presente trabalho é o resultado do trabalho do autor como formador de pinturas na Universidade de Artemisa. O objetivo deste trabalho é propor um sistema de ações de treinamento para aprimorar as habilidades dos diretores de escolas primárias rurais município de Bauta como principais professores de seus subordinados. Para o efeito, o treinamento foi assumido pela Universidade como uma atividade de pósgraduação no local de trabalho, a fim de favorecer o desenvolvimento da atividade pedagógica profissional de gestão no sistema de trabalho escolar. $\mathrm{O}$ uso de diferentes métodos, como pesquisa, entrevista, observação participante e análise documental, permitiu caracterizar o estado inicial, fundamentando a proposta e avaliando os resultados de sua aplicação, geralmente permitindo que os diretores assumam o papel de professor de seus subordinados.

Palavras-chave: Sistema de Ações, Treinamento, Atividade Pedagógica Profissional de Gestão e Escolas Primárias Rurais. 


\section{Introducción}

En la Educación Primaria se encuentran los escolares, cuyas edades constituyen una de las etapas fundamentales del desarrollo del ser humano. El Modelo de Escuela Primaria se sustenta en la idea de que:

Los cambios que se pueden lograr en materia de calidad de la educación están asociadas, esencialmente, al trabajo de la propia escuela, a la naturaleza de las transformaciones que se pretenden, a la interacción entre factores internos (directivos, maestros, alumnos) y factores externos (familia, comunidad) como agentes, estos últimos, que interactúan en los procesos educativos más cercanos al niño y a la escuela (Rico, Castillo, Silva, González, 2009, p. 6).

Los niños y niñas que residen en zonas rurales en Cuba, tienen las mismas oportunidades de acceder a una educación que favorezca su desarrollo integral al igual que los que residen en las grandes ciudades. Sin embargo para alcanzar esta aspiración la escuela primaria rural requiere contar con directores que posean las habilidades necesarias para el desarrollo de la actividad pedagógica profesional de dirección.

Las características del sector rural en el municipio de Bauta de la provincia de Artemisa, requiere de una atención priorizada en la preparación de sus directores que les permita perfeccionar la preparación de los docentes que laboran en estos centros.

El trabajo de los directores que tienen a su cargo la dirección institucional en las condiciones del rural demanda potenciar las habilidades directivas como principales maestros de sus subordinados (colectivo pedagógico), para que contribuyan a perfeccionar la organización escolar de acuerdo al Modelo actuante; que enseñen al colectivo pedagógico a organizar la vida de la escuela y que aprendan a aprovechar óptimamente los recursos humanos y materiales con que cuenta la escuela y la comunidad.

Aunque se han establecido estructuras organizativas en el país para el sector rural, las condiciones de cada territorio no son siempre iguales y no en todos existe la figura del director zonal. En el caso que nos ocupa cada una de las escuelas cuenta con un director, por lo que se hace necesario potenciar su papel como principal maestro de sus subordinados.

En Cuba el fin de la escuela primaria es contribuir a la formación integral de los estudiantes desde la primera infancia, constituyendo uno de los más significativos desafíos que los directores de las instituciones educativas deben enfrentar en la era del conocimiento donde 
las tecnologías de la informática y las comunicaciones enrumben en la sociedad.

Resulta necesario para los directores plantearse: ¿cómo preparar al colectivo pedagógico para lograr esta aspiración?, ¿qué objetivos deben lograrse en los alumnos?, ¿qué exigencias deben cumplir para alcanzar dichos objetivos?, ¿a qué escuela rural se aspira?,

Esto sitúa la atención al desarrollo de los docentes para llevar adelante las transformaciones de la enseñanza, como uno de los principales problemas que enfrentan los directores (as) en la aplicación de los conocimientos de Dirección Científica Educacional en la escuela rural, por cuanto la utilización de todas las potencialidades del colectivo pedagógico constituye una tarea de primer orden para lograr el desarrollo corriente y perspectivo de la institución educativa y por tanto del cumplimiento de los objetivos de dicha educación.

El cambio educativo es entendido como:

Aquellas transformaciones o modificaciones que tienen lugar en las concepciones educativas, y en los medios y recursos para llevarlos a cabo, incluyendo las que se deriven de su propia instrumentación (García, 1996, p. 5).

El cambio educativo se materializa por medio de la actividad pedagógica profesional de dirección (Alonso, 2000) que caracteriza y diferencia las funciones del director en relación al desempeño del docente, al actuar el primero, como principal maestro de sus subordinados.

Sin embargo no todos los directores han logrado incorporar en el sistema de trabajo de la escuela la actividad pedagógica profesional de dirección. En la base de este problema está, como una de las causas esenciales, el que un número nada despreciable de docentes acceden al cargo de director (a) sin la preparación previa en cuanto al dominio del contenido de la ciencia de la dirección; de las funciones a ejercer, entre las cuales, las vinculadas a este tipo de actividad, que constituye la esencia de su gestión en correspondencia con la responsabilidad asumida o por asumir, en un contexto, en muchas ocasiones, diferente al de donde provienen y se formaron.

Es por ello que no siempre la actividad pedagógica profesional de dirección, constituye, en la práctica, la esencia del proceso para la conducción del cambio educativo, lo que impacta negativamente en el desarrollo de las formas de existencia de la dirección: el proceso de dirección y la dirección de procesos, en particular este último, debilitado en su carácter desarrollador en la enseñanza aprendizaje de los alumnos 
como consecuencias de un insuficiente trabajo metodológico con y del colectivo pedagógico.

Sin embargo, en la revisión de la literatura disponible respecto a la actividad pedagógica profesional no se aborda con claridad lo relacionado con la dirección educacional. Se enfatiza más en la práctica de la dirección del proceso de enseñanza aprendizaje, lo que deja una brecha sin solucionar la referida a la preparación del directivo para su desempeño como principal maestro de sus subordinados directos.

Es (Alonso, 2002) el primero en Cuba en abordar el tema de la actividad pedagógica profesional de dirección. Este investigador demuestra, que el proceso de dirección de la institución educativa necesita ser fundamentado desde las ciencias pedagógicas, desde el análisis de sus categorías y de su utilización práctica en dicho proceso.

Aunque el autor, antes referido, en sus estudios llega hasta la definición de actividad pedagógica profesional de dirección situada en el universo de las diversas y complejas funciones de los directivos educacionales (la dirección escolar penetra todas las actividades que se realizan en la escuela, tanto con los alumnos, como con los trabajadores, familia y comunidad), y enfatiza en el carácter pedagógico de estas, que precisa de ellos un desempeño como principales maestros de sus subordinados directos, no aborda, por no ser objeto de su investigación, las estrategias de capacitación que contribuyan al desarrollo de las habilidades requeridas para ello.

Por otra parte, muchos de los directores (as) que asumen la responsabilidad, y una parte de sus superiores en las estructuras municipales, no jerarquizan el papel de la preparación y superación de los mismos como una vía pertinente y esencial de dar solución a los problemas teórico-prácticos del proceso de dirección que se desarrolla en la escuela.

A esto se le adiciona la insuficiente permanencia de los directivos de esta enseñanza en el cargo, lo que produce discontinuidad en los procesos de capacitación que se diseñan.

Además, se ha podido constatar que diversas acciones de capacitación (cursos, talleres y otras modalidades utilizadas) que se desarrollan en los municipios enfatizan más en lo académico, en el domino de documentos normativos $y$ en lo administrativo que en el desarrollo de las habilidades directivas indispensables para conducir la escuela de manera integral.

Son estas condiciones del desarrollo del trabajo de dirección en las escuelas primarias rurales las que aportaron la 
necesidad de diseñar y poner en práctica un sistema de acciones de capacitación que contribuyera a potenciar las habilidades de los directores (as) como los principales maestros de sus subordinados directos.

\section{Desarrollo}

\section{Aproximaciones teórico - metodológicas sobre la actividad pedagógica profesional de dirección}

Las funciones que los directivos de las escuelas primarias rurales desarrollan al frente de los colectivos pedagógicos y escolares están contenidas dentro de la actividad pedagógica profesional de dirección, la que constituye la esencia de su gestión.

La actividad pedagógica profesional de dirección potencia la elevación continua de la calidad de la educación: desde el perfeccionamiento del proceso de enseñanza aprendizaje (al garantizar, de manera contextual, el trabajo docente metodológico); profesionaliza a los docentes para que alcancen maestría pedagógica; promueve la labor científico investigativa en la solución de los problemas y desarrolla la atención a los cuadros subordinados y sus reservas en la dinámica de la escuela al lograr que la institución educativa se convierta en una:

Una escuela que aprende ... como ... un organismo que se autodesarrolla, se autorevisa, se autoconoce, se autorregula, se autodetermina y se autogestiona. Holly, P, citado por (Zilberstein, 2002, p. 7).

Por ello se hace necesario abordar la dirección desde una nueva concepción que posibilite la incorporación de manera activa de los sujetos del proceso docente educativo, aún cuando en la escuela existan carencias de docentes titulados y/o con experiencia para poder planificar, organizar, dirigir y controlar el mismo.

Esta dinámica del trabajo presupone demostrar en la práctica de la dirección de la escuela, todo el caudal de conocimientos y experiencias adquirida en la formación como docentes y en el ejercicio profesional previo a la asunción de la responsabilidad; y a la vez, en esa propia práctica, desarrollar habilidades para aprovechar las experiencias y conocimientos que cotidianamente construyen los docentes subordinados en un proceso de solución conjunta de tareas pedagógicas, tanto instructivas como educativa( García, 1996).

Si a todo lo anterior se le adiciona que la escuela tiene una diversidad de tareas que deben ser insertadas en la estrategia de trabajo metodológico con vistas a lograr, no sólo la preparación de los maestros y de sus estudiantes, sino de la familia y de la comunidad en su conjunto, se hace notorio el complejo papel 
que debe jugar el director dentro de la escuela primaria rural.

Es la escuela el escenario donde se desarrolla la actividad pedagógica profesional de dirección, eje de todos los procesos que acontecen en la misma, y donde el director (a), más que principal metodólogo y supervisor, es el principal maestro.

Desde ese punto de vista hay que valorar la presencia en el proceso de dirección de categorías pedagógicas como la enseñanza, la instrucción, entre otras, y el destacado valor de la Didáctica en dicho proceso. Así mismo, y siguiendo las ideas que al respecto expresa Alonso (2002), se debe tener en cuenta la educación en valores. Estos recursos científicos permiten concluir, entre otros importantes aspectos relacionados con el sistema de trabajo de los directivos de la escuela primaria rural, que estos requieren:

Fundamentar y ejecutar sus funciones, relaciones e interacciones con sus subordinados a partir de los principios y leyes ... de las Ciencias Pedagógicas y de la Educación (Alonso, 2002, p. 52).

Esto permitirá al directivo escolar fundamentar los procesos que se desarrollan en la escuela, y por tanto desplegar su papel como principal maestro de sus subordinados, en cuyo perfeccionamiento es protagónico su rol al frente de la institución educativa.

En la actividad pedagógica profesional de dirección se destacan conceptos como el de actividad, la que puede ser definida desde lo filosófico, psicológico, pedagógico y valorada desde la dirección como ciencia. De acuerdo con el Diccionario Filosófico La actividad es:
Una forma específicamente humana de relación activa con el mundo circundante se expresa como modo de ser del hombre y caracteriza la función e interacción del sujeto con el objeto, y la del sujeto con otros sujetos, y la relación del individuo con diferentes objetos, es estimulada por la necesidad .Se orienta hacia el objeto que da satisfacción a esta última y se lleva a cabo por medio de un sistema de acciones (Rosental \& Ludin, 2011, p. 4).

El desarrollo del proceso de dirección, incluye la definición de los objetivos, los medios, los resultados y el propio proceso; tiene carácter consciente e intencional para lograr la transformación del objeto y de si mismo.

Expresado en otros términos se precisa que la relación e interacción dirigente-subordinado, como actividad social, se comprenda como una relación donde uno y otro sean sujetos de la actividad, dotados de iniciativa $y$ creatividad en el proceso de las transformaciones de la enseñanza, de la 
institución y del crecimiento personal y profesional de sí mismos.

Precisamente en la actividad pedagógica profesional de dirección desarrollada por los directivos educacionales, está el revelar las necesidades de los subordinados y crear las motivaciones necesarias para asumir la sistemática formación, preparación y superación a través del trabajo metodológico, el que deben planificar, estructurar y poner en práctica en la escuela con el concurso de todos.

Como se señaló anteriormente, la dirección por su contenido tiene dos formas de existencia: el proceso de dirección y la dirección de procesos.

En el primer caso se evidencia las relaciones de subordinación dirigentes dirigidos, donde los primeros planifican, organizan, regulan y controlan la actividad de los segundos. Es un proceso funcional, propio de los directivos, el que se sustenta en los preceptos de la Dirección Científica Educacional.

En el caso de la dirección de procesos, expresa las funciones propias de los docentes, las que no comportan relaciones de subordinación entre el maestro o profesor y los educandos. Esa dirección de procesos es planificada, organizada, regulada y controlada por los profesionales que la ejecutan, quienes recurren teórica y metodológicamente a la Pedagogía y las Ciencias de la Educación sobre todo a la Didáctica para su conducción.

En conclusión, en el proceso de dirección se pone de manifiesto las relaciones de subordinación y de cooperación entre el directivo escolar y sus subordinados con el objetivo de proporcionarle los recursos pedagógicos, didácticos y materiales necesarios, a partir de la atención a sus diferencias individuales, con vistas a desarrollar su personalidad y profesionalidad, en función de los objetivos de la educación.

Esto alimenta la idea de que la gestión de dirección pueda concebirse como un sistema de acciones donde se ponen en práctica los conocimientos y la experiencia que se posee previamente y se concretan métodos y procedimientos para el aprendizaje continuo.

En este sentido Carlos Manuel Álvarez de Zayas plantea:

Para la actividad lo fundamental es el motivo; para la acción, el objetivo; y para la operación, las condiciones (Álvarez, 1996, p. 69).

Las acciones y las operaciones, integran un sistema que forman parte de la estructura de la actividad que se produce entre el hombre y la realidad objetiva que lo rodea, mediante el cual satisface sus 
necesidades, transforma la realidad y a sí mismo.

La actividad pedagógica profesional de dirección contiene en su esencia la actividad de dirección la que se caracteriza por la especialización de los dirigentes en orientar el trabajo colectivo, influyendo sobre los subordinados, de manera consciente, premeditada, sistemática y estable, a fin de lograr los objetivos propuestos.

También en su contenido, esta actividad integra la categoría actividad pedagógica profesional, definida por el DrC. Lisardo García del Instituto Central de Ciencias Pedagógicas de la República de Cuba en 1992 y citada por (Alonso, 2002, p. 45) con los siguientes términos:

Es aquella actividad que está dirigida a la transformación de la personalidad de los escolares en función de los objetivos que plantea el Estado a la formación de las nuevas generaciones. Se desarrolla en el marco de un proceso de solución conjunta de tareas pedagógicas, tanto de carácter instructivas como educativas y en condiciones de plena comunicación entre el maestro, el alumno, el colectivo escolar y pedagógico, la familia y las organizaciones estudiantiles.

Retomando el estudio realizado por Alonso se puede concretar que:

Aunque esta categoría se ha reducido hasta ahora al marco de la relación "educador-educando", se valoró que - dada la amplitud de su contenido - podría extenderse también a la relación "dirigentedirigido (Alonso, 2002, p. 45).

El DrC. Alonso, en el trabajo citado en párrafos anteriores, concluye que la actividad pedagógica profesional de dirección:

Es una forma de actividad de dirección específica del sector educacional, que se distingue por el marcado carácter técnicometodológico y científicopedagógico con que se desarrolla el proceso de dirección y por su clara orientación hacia la transformación de la personalidad y el desarrollo profesional de los cuadros y docentes, en función de los objetivos que plantea el Estado a la formación de las nuevas generaciones; actividad que se desarrolla en el marco de un proceso de solución conjunta de tareas pedagógicas - tanto instructivas como educativas -, y en condiciones de plena comunicación entre dirigentes y dirigidos, con la activa participación de las organizaciones políticas, sociales y de masas que actúan en su entorno (Alonso, 2002, p. 46).

La definición asumida por la autora conlleva, que el director (a) se reconozca, se sienta y actúe como maestro de sus subordinados directos, de los cuales también aprende. Esto implica ver, desde lo integral, la interrelación de varios elementos esenciales que contribuyen a la elevación de la calidad de la educación: la gestión de dirección, (que a criterio de la 
autora no es sólo prestar atención a la administración de los recursos materiales y financieros, sino, esencialmente, el uso del conocimiento y la experiencia en estos aspectos y al trabajo docente.

Indica que en el desarrollo del trabajo metodológico el director(a) debe garantizar la profesionalización de sus docentes (y colaboradores) y la formación de su personalidad en correspondencia con los objetivos que el país ha situado para la formación del ciudadano que vivirá en la sociedad en tanto contribuye a su edificación.

En esencia, las posibilidades de eficiencia docente educativa que demuestren los docentes e incluso los restantes directivos de la institución educativa, en función de la formación integral de los educandos, dependerá en gran medida, de la influencia que el director (a) ejerza en los subordinados para lograr las transformaciones de la personalidad de estos y en el desarrollo de su profesionalidad, lo que contribuye al mejoramiento humano de los mismos y al desarrollo de la institución.

La profesionalización como proceso tiene como esencia:

La especialización de los recursos laborales calificados, a través del cual se logra alcanzar la eficiencia en la adquisición y/o desarrollo de las competencias básicas exigidas por el modelo profesional y las aspiraciones individuales (Añorga, 2002, p. 11).

Las posibilidades que tiene el director (a) de la escuela primaria rural de la transformación de la personalidad y el mejoramiento profesional y humano de los subordinados parte del dominio que este tenga respecto de la incorporación a su actuación como principal maestro, de los procesos cognitivos y afectivos, desarrollo de valores e introducción de conocimientos, mediante la acción participativa de los sujetos y de si mismo sin perder de vista el desarrollo de las motivaciones del colectivo.

Asumirse como principal maestro de sus subordinados directos, es reconocer, como función de dirección, lo que en la década de los 80 definía Celia Pérez, autora del libro "Hacia el perfeccionamiento del trabajo de la dirección escolar" al expresar que:

Dirigir equivale a organizar, orientar, ayudar, encaminar, guiar, enseñar cómo hacer una cosa de la mejor forma, observar, penetrar en la esencia del asunto, aconsejar, encontrar las deficiencias y buscar las vías para erradicarlas, detectar lo bueno, lo mejor, mantenerlo, superarlo, apoderarse de la mejor experiencia e incorporarla al bagaje de dirección, interesar a los demás en el trabajo, consultar con los demás, revisar, chequear, supervisar, controlar a aquellos que 
llevan a cabo un trabajo, evaluar, estimular (Pérez, 1983, p. 25).

De esa manera esta autora dejaba sentada algunas de las principales habilidades $\quad \mathrm{y} \quad$ conocimientos indispensables para asumir la dirección de una institución educativa, a la vez que evidenciaba la importancia determinante del director(a) de escuela en el proceso de dirección, donde su influencia tanto en el plano individual como colectivo hace significativos aporte al desarrollo de la personalidad y en lo profesional de sus subordinados al:

Potenciar el perfeccionamiento del colectivo de la institución en el proceso del cambio educativo (transformaciones);

$\checkmark \quad$ desarrollar sus conocimientos, habilidades, capacidades y actitudes individuales (valores) que contribuyan al enriquecimiento de la cultura de la institución educativa;

garantizar el cumplimiento de los objetivos trazados (Estrategia, Convenio Colectivo de Trabajo, Plan de Trabajo Individual, entre otros, recogidos en el proyecto educativo de la institución).

Acciones todas que en el escenario de la escuela primaria rural nos pone en presencia de una concepción desarrolladora de la dirección, la que se caracteriza por:
* atender al hombre como objeto y sujeto de su propia dirección;

*aprender dirigiendo y dirigir enseñando;

* privilegiar el trabajo en equipo para corresponder a los intereses colectivos y sociales;

* desarrollar la comunicación con un carácter dialógico;

* aplicar un enfoque personológico que permita dirigir con y no sobre las personas;

* dar sentido real a la participación de los integrantes del colectivo pedagógico y escolar en las decisiones;

* hacer del proceso de dirección un instrumento metodológico de auto aprendizaje y sistematización de la realidad docente educativa.

Lo expresado sugiere que cualquier gestión de dirección debe comenzar por el diagnóstico de la institución educativa de manera integral con énfasis en los recursos humanos, lo que permitirá contextualizar la actividad pedagógica profesional de dirección como una función.

Es evidente que para lograr el efecto deseado en la ejecución de la actividad pedagógica profesional de dirección se hace indispensable alcanzar el desarrollo de determinadas habilidades que les permita a los (las) directores (as) ejercer sus funciones de manera efectiva $y$ 
continuamente perfeccionada en las transformaciones de la personalidad de sus subordinados.

Porque asumir una responsabilidad de dirección sin evaluar previamente en qué medida los conocimientos, habilidades, hábitos que se poseen, responden o no a los requisitos del cargo a ocupar, a las funciones específicas que hay que cumplir, al modelo actuante y al proyectivo de escuela es como diseñar el fracaso.

Las habilidades son un componente esencial $\mathrm{y}$ decisivo en el modo de actuación de cualquier profesional, pero en particular, en los de la educación. La habilidad:

Constituye el dominio de las operaciones (psíquicas y prácticas) que permiten la regulación de la actividad. Es la comprensión de la interrelación entre el fin de la actividad y las condiciones, los medios de su puesta en práctica (Ferrer, 2004, p. 79).

Se concuerda con Ferrer ya que esta definición posibilita enriquecer los argumentos que se han estado utilizando para sustentar la labor de principal maestro de los subordinados directos por parte de los directivos de las escuelas primarias rurales en el desarrollo de la actividad pedagógica profesional de dirección como contenido del proceso de dirección.
En la práctica de la gestión de dirección los directivos escolares deben adquirir paulatinamente, el dominio de las operaciones indispensables para la realización del trabajo metodológico, hasta lograr un significativo nivel de calidad en la ejecución de las transformaciones en la institución educativa.

Pero la adquisición y dominio de las habilidades indispensables para la realización de las acciones y operaciones como principal maestro de los subordinados directos, implica adquirir conocimientos, con énfasis en Dirección Científica Educacional que contribuyan a la realización con mayor eficiencia de sus funciones tanto específicas como generales, y a aplicar acertadamente los componentes de orientación, ejecución y de control de las actividades.

Lo anterior concuerda con el criterio de que la habilidad es el conocimiento en la acción, es saber hacer y está en correspondencia con las funciones de planificación, organización, regulación y/o ejecución y de control que caracteriza a una dirección eficiente.

Las habilidades que se adquieren en la práctica directiva se sustentan en las desarrolladas durante los estudios de pregrado y puestas en práctica en la vida profesional al frente de grupos de alumnos. Es decir, que se trata de integrar 
conocimientos, habilidades y hábitos previamente desarrollados para ponerlos en función de la gestión directiva como principal maestro de los subordinados $\mathrm{y}$ desde ellos dar entrada a los nuevos saberes, internalizarlos.

Tener conciencia de este proceso ayuda a entender que el proceso de dirección:

Está caracterizado, y se añade que está condicionado, por la interrelación dialéctica entre los objetivos que se persigan para el logro de las tareas dentro del proceso (docente educativo) y la transformación de los modos de actuación como la expresión más general del cumplimiento de los objetivos (Ferrer, 2004, p. 81).

Las aproximaciones teóricas realizadas por la autora de este trabajo a los procesos de autodesarrollo/autoeducación y procesos autógenos, la motiva a buscar vías que posibiliten el desarrollo de la personalidad desde sí mismo. Por ello asume la propuesta de (Fariñas, 1995) en su texto "Maestro una estrategia para la enseñanza", de Habilidades Conformadoras del Desarrollo Personal las que por su grado de generalización y poder autorregulador del desarrollo integral de la personalidad, pueden constituirse en el objetivo formativo de preparación y de superación de los directivos escolares de las escuelas primarias rurales como principales maestros de sus subordinados directos:

Fariñas argumenta que estas habilidades dan una amplia perspectiva al crecimiento de la personalidad, del yo, tanto en un sentido cuantitativo como cualitativo, porque le permiten su desarrollo en una forma constructiva, independiente o autodidáctica, lo que es realmente una vía aceptable para quienes enfrentan la dirección de la institución educativa sin la preparación previa y sin el tiempo necesario para adquirir por las vías formales los conocimientos, habilidades, hábitos y métodos para la conducción de personas.

Las Habilidades Conformadoras del Desarrollo Personal definidas por la autora antes mencionada, son cuatro:

*I Habilidades relacionadas con el planeamiento y consecución de metas personales, y con la organización temporal general de la vida cotidiana.

* II Habilidades relativas a la comprensión y búsqueda de información.

*III Habilidades relativas a la comunicación y a la relación con los demás.

* IV Habilidades relacionadas con el planteamiento y solución de problemas.

Estas habilidades responden, en el tiempo, a los pilares de la educación para el siglo XXI trazados en el Informe Delors 
de la UNESCO (1996), los que se sintetizan en las siguientes ideas de autodesarrollo:

* aprender a ser;

* aprender a aprender;

* aprender a vivir juntos y a

* aprender a hacer.

Tomando en consideración la propuesta asumida, los procesos de formación, preparación y superación de los directores (as) de las escuelas primarias rurales tienen que contribuir a la potenciación de esas habilidades para influir conscientemente sobre sí mismos y, al mismo tiempo, aprovechar y promover el desenvolvimiento de sus colaboradores.

Pudiera ejemplificarse con la siguiente propuesta: el director (a) debe auto diagnosticar sus estrategias de aprendizaje para concluir los aspectos fuertes y débiles de las mismas, de manera que llevadas a discusión con sus subordinados pueda convertir los elementos fuertes, de carácter personal, en conocimientos trasmisibles a los mismos, en la medida en que estos contribuyen a la eliminación o corrección de las estrategias fallidas.

Sus experiencias se convierten en fuente de aprendizaje relevante, aún cuando algunas actúan como barreras para el cambio.
Por lo tanto, la tarea de los profesores de la Facultad de Ciencias de la Educación de la Universidad de Artemisa, es ayudar a tomar conciencia de la necesidad de conocer y aprender. Este es el punto inicial para emprender cualquier acción de superación de postgrado.

La primera acción fue explorar los conocimientos que tenían los directores(as) en relación con el diagnóstico de su institución educativa, para poder iniciar el sistema de acciones a partir de las necesidades y motivaciones de los propios interesados y sujetos del cambio.

\section{Fundamentos de la propuesta}

Esta experiencia se desarrolló en el municipio de Bauta, con directores de escuelas primarias rurales durante un curso de postgrado desarrollado por la Facultad de Ciencias de la Educación de la Universidad de Artemisa, Cuba en el curso escolar 2015-2016.

El diseño y desarrollo del sistema de acciones para potenciar las habilidades de los directores (as) de las escuelas primarias rurales como principales maestros (as) de sus subordinados, lleva implícito el mejoramiento continuo de su actividad profesional de dirección y para su aplicación se tuvo en cuenta varios 
presupuestos caracterizadores del sistema, entre ellos:

- El sistema de trabajo de la escuela como un sistema abierto y autogobernado;

- Coherencia de las actividades en diversos espacios y niveles de la estructura con enfoque de sistema y de manera sistemática;

- Las potencialidades y debilidades del colectivo pedagógico y del propio director (a);

- Flexibilidad;

- Pertinencia;

- Propiciar el aprender de los errores;

- Énfasis en el autodesarrollo;

- Reconocimiento de la diversidad;

- Aprovechamiento de la experiencia institucional y personal;

- Relación teoría práctica con respeto al contexto.

\section{Aspectos que constituyeron elementos integradores del sistema de acciones}

* La utilización de métodos científicos para la conducción de las transformaciones.

* Técnicas e instrumentos para el diagnóstico integral con énfasis en los recursos humanos.

* La unidad y coherencia de acciones metodológicas de la universidad y la escuela.
* La capacitación de todos los factores que influyen en el funcionamiento de la escuela con énfasis en los directores de escuelas y las reservas.

Desde los referentes pedagógicos se asumió la necesidad de superar la enseñanza tradicional por un modelo más vinculado a una enseñanza humanista, tutoreal, personalizada, que preste atención priorizada al desarrollo integral de la personalidad de los directivos, a los aspectos formativos vinculados a enseñar a aprender, enseñar a hacer y enseñar a ser, a cada persona, más que a los aspectos puramente teóricos de la dirección, y que lo hagan desde un acercamiento de los cuadros superiores a la realidad en que se encuentra insertado el subordinado directo, y el conjunto de subordinados como un todo, de las motivaciones e intereses, y de las capacidades que estos posean (Zona de Desarrollo Próximo), asociado a un proceso de transformación de los mismos, de su preparación y de sus concepciones de la dirección del proceso docente educativo.

Desde el punto de vista metodológico se asumió, dentro del sistema de acciones, la enseñanza de cuadros como una implicación de compromiso, de involucrarse, de interdependencia, interacción e interés común (Doménech, 2002), donde la capacitación se dirige teniendo como base 
la propia experiencia de los participantes, para propiciar cambios en su comportamiento, es decir, en el pensar y accionar, para transformar el contexto.

Implicó comprender la capacitación como actividad, como proceso de interacción - transformación; dirigida a la solución de problemas o situaciones como base que estimula el aprendizaje; a la vez, que se propició la motivación, el compromiso e identificación con lo que se aprende.

Por ello, la propuesta de capacitación, independiente de sus características intrínsecas, abordó el contenido con una visión interdisciplinaria y holística. No puede olvidarse que la dirección como ciencia se apoya en diversas ciencias sociales, humanistas $\mathrm{y}$ exactas, incluida la informática, pero con un enfoque eminentemente pedagógico.

Los cuadros en ejercicio y/o reservas que participaron en las actividades de capacitación (formación, preparación y superación) tienen experiencias y creencias relativas, adquiridas en el medio en que se desempeñan, por lo que fueron adiestrados en acciones que le permitiera evitar $y$ defenderse de aquellas que no contribuyan a realizar sus funciones con eficiencia.

Sistema de acciones para potenciar las habilidades del director (a) como principal maestro de sus subordinados
El sistema de acciones comprendió cuatro etapas de trabajo:

Primera etapa: de sensibilización y diagnóstico

Se caracterizó por la discusión con los directores y sus reservas de las escuelas primarias rurales, acerca de las definiciones conceptuales y la necesidad de mejorar sistemáticamente el proceso de dirección, la dirección de procesos, el trabajo metodológico, la organización escolar y el papel de los cuadros como principales maestros de sus subordinados directos en el ejercicio de la Actividad Pedagógica Profesional de Dirección.

Se realizó un diagnóstico de cada cuadro o directivo escolar y sus reservas a partir de:

$\checkmark$ resultados de sus evaluaciones,

$\checkmark \quad$ resultados de las visitas recibidas y efectuadas,

$$
\checkmark \text { Criterios de los }
$$
subordinados directos y jefes superiores y

$\checkmark \quad$ La autopercepción sobre sus conocimientos y habilidades para dirigir.

$$
\text { Se aplicaron encuestas, }
$$
entrevistas, observaciones y otros métodos empíricos y teóricos de investigación.

Segunda etapa: de definición grupal y metodológica

Se caracterizó por la ubicación en grupos afines de acuerdo al nivel de 
desarrollo alcanzado y demostrado tanto en el orden teórico como práctico.

Se diseñaron acciones para la atención diferenciada a cada sujeto, los enfoques esenciales para el trabajo con las personas y las modalidades a utilizar.

Se definieron los temas a trabajar en el postgrado y las actividades prácticas a desarrollar, lográndose coherencia en las acciones en relación a los objetivos previamente acordados, de manera que el mismo tema fue abordado por diversas vías en forma de cascada y se concretó en el puesto de trabajo como parte del sistema de trabajo.

Tercera etapa: del desarrollo de las acciones para el mejoramiento de la actividad pedagógica profesional de dirección.

Se caracterizó por la ejecución de las acciones de capacitación ajustadas a las necesidades de los contenidos básicos para el desempeño de las funciones de dirección.

Entre los contenidos básicos para el desempeño de sus funciones se abordaron:

* Aspectos esenciales de la organización escolar;

* El sistema de trabajo de la escuela y dentro de este el funcionamiento de los órganos técnicos y de dirección incluido el Consejo de Escuela;
* Documentos normativos de la Política Educacional, del trabajo metodológico y del modelo pedagógico de la enseñanza y el de la universalización;

* Contenidos básicos y didácticos;

* Métodos para la realización del diagnóstico integral que le permitan conocer y actuar de acuerdo a las necesidades de sus docentes y directivos subordinados;

* Métodos y técnicas básicas de dirección y de la administración de recursos., entre ellas la delegación de autoridad; la toma de decisiones efectivas, técnicas de trabajo con grupos; técnicas del control con énfasis en el control interno; funciones generales de dirección; solución de problemas y conflictos; entre otras;

* Métodos para el desarrollo de la metacognición;

* Concepción del trabajo metodológico para lo que fue necesario enfatizar en cómo:

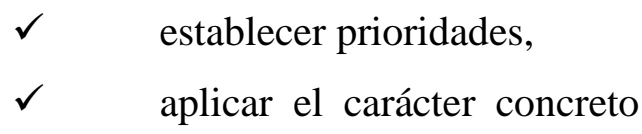
y diferenciado del contenido en función de los problemas y necesidades colectivas e individuales,

$\checkmark \quad$ integrar saberes filosóficos,
psicológicos, sociológicos, políticos,
científicos-teóricos y pedagógicos en el
contenido de la dirección,


desarrollar el enfoque sistémico, teniendo en cuenta el carácter rector de los objetivos,

evaluar el desempeño con un carácter diferenciado, ejecutar la entrega pedagógica,

$\checkmark \quad$ diseñar $\quad$ y aplicar comprobaciones de conocimiento y a trabajar con los resultados de estas,

usar los indicadores de eficiencia como herramientas para la toma de decisiones,

controlar la superación de los docentes y

$\checkmark \quad$ trabajar con las organizaciones del centro y de la comunidad.

Cuarta etapa: del control y la evaluación

Se caracterizó por acciones significativas como:

$\checkmark \quad$ Discusión y análisis de los resultados de las visitas que recibían y realizaban en la escuela;

Observación y valoración de los modos de actuación que asumían ante diversos problemas y distintos escenarios;

$\checkmark \quad$ Observación de los modos de actuación y dominio que demuestra del contenido y de los métodos en las actividades que dirige y/o en las que participa en su contexto o fuera de este;
Valoración del modo en que ponen en práctica de manera creativa lo aprendido;

$\begin{array}{lll}\checkmark & \text { Resultados } & \text { favorables } \\ \text { logra en los puntos medulares del } \\ \text { desarrollo institucional. }\end{array}$

\section{Resultados obtenidos}

A través de la observación participante, revisión de documentos y encuestas de satisfacción se pudo constatar el mejoramiento continuo de la Actividad Pedagógica Profesional de Dirección por la apropiación de métodos y estilos de trabajo coherentes que pusieron en práctica en sus modos de actuación; el mejoramiento continuo de la capacidad de dirección de los directivos y docentes; el fortalecimiento de la organización escolar y las relaciones con el entorno (familia y comunidad); el mejoramiento del trabajo metodológico y su expresión en la calidad de las clases; el incremento en el aprendizaje de los alumnos, así como el perfeccionamiento del proceso de evaluación del escolar y de los docentes incluyendo al director.

El sistema de acciones contribuyó a la formación de una dirección desarrolladora, poniéndose de manifiesto de manera activa y participativa, donde primaron los valores compartidos. 
En el sistema de acciones se tuvo en cuenta la importancia del papel protagónico del hombre como objeto $\mathrm{y}$ sujeto de la dirección; así como del estudio, de la preparación y superación de los directores de las escuelas primarias rurales.

Por otra parte, se abrieron posibilidades para el uso de las Tecnologías de la Informática y las Comunicaciones (Tics), lo que tiene un valor operacional significativo, que condiciona la necesidad de que por las diversas vías del trabajo metodológico y, en particular por las visitas de ayuda metodológica (VAM), se capacite a los directivos escolares y sus reservas en el uso de ellas de manera que puedan hacer una utilización eficiente en las funciones básicas de:

1. Producir y/o seleccionar los materiales básicos para su auto aprendizaje $\mathrm{y}$ diseñar las estrategias y secuencias de aprendizajes para el logro de las competencias específicas y generales para la dirección del modelo pedagógico de su enseñanza conjuntamente con logros, conocimientos, habilidades, valores $\mathrm{y}$ actividades previstas como aseguramiento a lo diseñado por la Universidad y por la propia institución.

2. Gestionar la información y los conocimientos para el aprendizaje de sus subordinados, en ambiente digitales más abiertos, y preparar al mismo tiempo a sus docentes en estas habilidades.

3. Gestionar los aprendizajes personalizados a partir de las características de cada uno de sus docentes.

\section{Fuentes de verificación y control}

Estos resultados pudieron comprobarse a través de las visitas de ayuda metodológica (VAM) e inspección con énfasis en el seguimiento a la capacitación; en el análisis sistemático en los órganos técnicos y de dirección de los indicadores de eficiencia de la institución educativa; en los resultados de los cortes evaluativos del personal docente y de los cuadros y en la recogida del estado de opinión de docentes, trabajadores, estudiantes y familia, así como de las autoridades educacionales.

\section{Conclusiones}

Se argumenta que la actividad pedagógica profesional de dirección constituye el contenido esencial de la gestión de los directores de las escuelas rurales. Su adecuado desarrollo a través del sistema de trabajo contribuye a garantizar la materialización de la concepción de la escuela. 
La propuesta presentada aplicada en escuelas rurales del municipio de Bauta, provincia de Artemisa tributa al perfeccionamiento de la dirección educacional enfatizando en el papel del director como principal maestro de sus subordinados, en la atención de las potencialidades de los maestros para llevar adelante las transformaciones que se realizan en la escuela cubana actual.

Los resultados alcanzados en el proceso docente educativo, en los centros donde se aplicó la propuesta, evidencian, que en la práctica de la gestión de un significativo número de instituciones educativas, la actividad pedagógica profesional de dirección, no constituía la esencia del proceso para la conducción del cambio educativo.

El desarrollo del sistema de acciones contribuyó a mejorar la Actividad Pedagógica Profesional de Dirección, en su despliegue a través del sistema de trabajo; aportó posibilidades y conocimientos para el perfeccionamiento de los métodos que emplean tanto en su interacción, como en la influencia que ejercen en el personal docente y los alumnos; ayudó desde la práctica a integrar procesos que generalmente se desarrollan en la práctica aislados como: el trabajo docentemetodológico y el científico-metodológico, la superación del personal docente y la gestión de la dirección educacional.

El sistema de acciones va encaminado a dar respuesta a la evidente necesidad de la interrelación entre desarrollo y capacitación con énfasis en los conocimientos, habilidades y hábitos de los directores(as) y sus reservas en las escuelas primarias rurales, con el fin de prepararlos para que desempeñen adecuadamente sus funciones en su puesto de trabajo como los principales maestros de sus subordinados.

\section{Referencias}

Alonso, S. H. (2002). El Sistema de Trabajo del MINED. (Tesis en Educación). Instituto Central de Ciencias Pedagógicas, Habana.

Alvarez, C. M. (1995). La escuela en la vida. La Habana: Editorial Pueblo y Educación.

Añoga, J. (2002). Aprender y enseñar en la escuela. La Habana: Editorial Pueblo y Educación.

Delors, J. (1996). La educación encierra un tesoro. Madrid: Ediciones UNESCO.

Domenech, S. (2002). Cuba: economía en el Período Especial. La Habana: Editorial Política.

Fariñas, G. (1995). Maestro una estrategia para la enseñanza. La Habana: Editorial Academia.

Ferrer, M. T. (2004). Las Habilidades Pedagógicas Profesionales en el Maestro 
Primario. Modelo para su Evaluación. La Habana: Editorial Pueblo y Educación.

Garcia, L. (1996). Los retos del cambio educativo. La Habana: Editora Pueblo y Educación.

Pérez, C. (1983). Hacia el perfeccionamiento de la escuela primaria. La Habana: Editorial Pueblo y Educación.

Rosental, M., \& Ludin, P. (2011). Diccionario Filosófico. La Habana: Editorial Pueblo y Educación.

Rico, P., Castillo, S., Silva, R., \& González, R. A. (2009). Modelo de escuela primaria; principales transformaciones. En Curso 3 Evento Pedagogía Internacional, Cuba.

Zilberstein, J. (2002). Diagnóstico y transformación de la Institución docente. México: Ediciones CEIDE.

Recebido em: 20/06/2017 Aprovado em: 20/08/2017 Publicado em: 10/11/2017

\section{ORCID}

Maria Julia Aguilar Aguilera

http://orcid.org/0000-0002-4064-5423

\footnotetext{
Como citar este artigo / How to cite this article / Como citar este artículo:

APA:

Aguilera, M. J. A. (2017). Los directores(as) de las escuelas primarias rurales como los principales maestros de sus subordinados. Rev. Bras. Educ. Camp., 2(2), 728-749. DOI: http://dx.doi.org/10.20873/uft.25254863.2017v2n2p728

ABNT:

AGUILERA, M. J. A. Los directores(as) de las escuelas primarias rurales como los principales maestros de sus subordinados. Rev. Bras. Educ. Camp., Tocantinópolis, v. 2, n. 2, p. 728-749, 2017. DOI: $\quad$ http://dx.doi.org/10.20873/uft.25254863.2017v2n2p728
} 